OPEN ACCESS

Edited by:

Weiwen Zhang,

Tianjin University, China

Reviewed by:

Kesen Ma,

University of Waterloo, Canada Xu Fang,

Shandong University, China Suping Yang,

Huaqiao University, China

*Correspondence:

Xuefeng Lu

Ivxf@qibebt.ac.cn

Specialty section:

This article was submitted to Microbial Physiology and Metabolism, a section of the journal

Frontiers in Microbiology

Received: 02 February 2016

Accepted: 15 April 2016

Published: 29 April 2016

Citation:

Wang M and LuX (2016) Exploring

the Synergy between Cellobiose

Dehydrogenase from Phanerochaete chrysosporium and Cellulase from

Trichoderma reesei.

Front. Microbiol. 7:620.

doi: 10.3389/fmicb.2016.00620

\section{Exploring the Synergy between Cellobiose Dehydrogenase from Phanerochaete chrysosporium and Cellulase from Trichoderma reesei}

\author{
Min Wang ${ }^{1,2}$ and Xuefeng Lu ${ }^{1,3 *}$ \\ ${ }^{1}$ Key Laboratory of Biofuels, Qingdao Institute of Bioenergy and Bioprocess Technology, Chinese Academy of Sciences, \\ Qingdao, China, ${ }^{2}$ Shandong Provincial Key Laboratory of Energy Genetics, Qingdao Institute of Bioenergy and Bioprocess \\ Technology, Chinese Academy of Sciences, Qingdao, China, ${ }^{3}$ Shandong Provincial Key Laboratory of Synthetic Biology, \\ Qingdao Institute of Bioenergy and Bioprocess Technology, Chinese Academy of Sciences, Qingdao, China
}

Recent demands for the production of lignocellulose biofuels boosted research on cellulase. Hydrolysis efficiency and production cost of cellulase are two bottlenecks in "biomass to biofuels" process. The Trichoderma cellulase mixture is one of the most commonly used enzymes for cellulosic hydrolysis. During hydrolytic process cellobiose accumulation causes feedback inhibition against most cellobiohydrolases and endoglucanases. In this study, we demonstrated the synergism effects between cellobiose dehydrogenase $(\mathrm{CDH})$ and cellulase both in vitro and in vivo. The $\mathrm{CDH}$ from Phanerochaete chrysosporium was heterologously expressed in Pichia pastoris. Supplementation of the purified $\mathrm{CDH}$ in Trichoderma cellulase increased the cellulase activities. Especially $\beta$-glucosidase activity was increased by $30-100 \%$ varying at different time points. On the other hand, the cdh gene was heterologously expressed in Trichoderma reesei to explore the synergism between $\mathrm{CDH}$ and cellulases in vivo. The analyses of gene expression and enzymatic profiles of filter paper activity, carboxymethylcellulase (CMCase) and $\beta$-glucosidase show the increased cellulase activity and the enhanced cellulase production in the $c d h$-expressing strains. The results elucidate a possible mechanism for diminishing the cellobiose inhibition of cellulase by $\mathrm{CDH}$. These findings provide a novel perspective to make more economic enzyme cocktails for commercial application or explore alternative strategies for generating cellulase-producing strains with higher efficiency.

Keywords: Tritroderma reesei, cellulase, cellobiose inhibition, cellobiose dehydrogenase, synergy

\section{INTRODUCTION}

The filamentous fungus Trichoderma reesei produces a set of enzymes that cooperate synergistically in the degradation of cellulose. They are nowadays a paradigm for commercial scale production of cellulases and employed for the saccharification of cellulose biomass to simple sugars for biofuel production. The widely accepted mechanism for enzymatic cellulose hydrolysis involves synergistic actions by endoglucanase (EGs) (EC 3.2.1.4), exoglucanase (cellobiohydrolases, CBHs, in the case of T. reesei) (EC 3.2.1.91; EC 3.2.1.176), and $\beta$-glucosidases (BGLs) (EC 3.2.1.21) (Zhang and Lynd, 2004). Endoglucanases randomly hydrolyze accessible intramolecular $\beta$-1,4-glucosidic bonds of cellulose chains to produce new chain ends; exoglucanases processively cleave cellulose 
chains at the ends to release soluble cellobiose or glucose; and $\beta$-glucosidases hydrolyze cellobiose to glucose. These three hydrolysis processes occur simultaneously.

The mechanism of induction and repression on cellulase stimulated by hydrolysates formed from cellulose hydrolysis has been explored in depth (Schmoll and Kubicek, 2003). In most cases, the production and/or activity of cellulase components are repressed or inhibited by the hydrolysis products (Holtzapple et al., 1990; Ju and Afolabi, 1999). It is well established that cellobiose with high concentration inhibits the activity of cellulase (George et al., 2001). It is a stronger inhibitor than glucose (Holtzapple et al., 1990). Accumulation of cellobiose causes feedback inhibition against most cellobiohydrolases and endoglucanases, and thus it becomes important to supply enough $\beta$-glucosidases to hydrolyze cellobiose to glucose in order to eliminate such inhibition.

In $T$. reesei cellulase system, the two most abundant proteins are cellobiohydrolases CBH I and CBH II, which are known to account for 70 to $80 \%$ of the total cellulases (Nummi et al., 1983). The other abundant component is endoglucanase EG II (CEL5A) with up to $15 \%$. $\beta$-glucosidases is relatively scarce in $T$. reesei. A concept of designing a highly efficient cellulase mixture for hydrolysis of cellulose has been reported (Gusakov et al., 2007), and inspired many efforts to improve cellulase performance by making the cocktails with supplementary $\beta$-glucosidases and/or other components (Knauf and Moniruzzaman, 2004). $\beta$-glucosidases from Aspergillus niger has been purified and manufactured as the commercial Novozyme 188 (Novozymes), which is one of the most commonly used enzyme for promotion of a synergistic effect with Trichoderma cellulases. The cocktail formulation supplemented with this BGL can efficiently produce fermentable glucose and the hydrolytic efficiency was increased by enhancing the $\beta$-glucosidase activity in the enzyme cocktail (Ma et al., 2011; Dashtban and Qin, 2012; Nakazawa et al., 2012). The synergism between $A$. niger $\mathrm{BGL}$ and $T$. reesei cellulase has triggered interest in screening and discovery other promise enzymes to create more effective and balanced commercial cellulase mixtures (Park et al., 2012).

Cellobiose dehydrogenase (CDH, EC 1.1.99.18) is an extracellular enzyme produced by various wood-degrading fungi (Henriksson et al., 2000). It oxidizes the reducing end of cellobiose and cellooligosaccharides to their corresponding 1, 5-lactones, which are subsequently hydrolyzed to the carboxylic acids. Early in 1978, Ayers suggested that CDH (named as cellobiose oxidase, CBO back then) from Sporotrichum pulverulentum (Phanerochaete chrysosporium) might be involved in cellulose biodegradation (Ayers et al., 1978). Bao and Renganathan (1992) reported that CBO which was purified from extracellular medium of Phanerochaete chrysosporium could enhance crystalline cellulose degradation by $T$. viride and $T$. reesei cellulases, but the mechanism remained unclear. Igarashi demonstrated the synergistic interaction between $\mathrm{CDH}$ and $\mathrm{CBH}$ I from $P$. chrysosporium (Igarashi et al., 1998). Decomposition of bacterial cellulose microcrystalline by $\mathrm{CBH}$ I was enhanced by the combined addition of $\mathrm{CDH} /$ ferricyanide. The reason was clarified that $\mathrm{CDH}$ could relieve the competitive inhibition of cellobiose to $\mathrm{CBH}$ I by its oxidation to cellobionolactone. The synergism between $\mathrm{CDH}$ and $\mathrm{CBH}$ I observed in P. chrysosporium inspired our interests in application of this synergism in $T$. reesei cellulase system.

The synergism of $\mathrm{CDH}$ from another wood-rotting basidiomycete, Pycnoporus cinnabarinus, with $T$. reese $i$ industrial enzyme cocktail in the saccharification process on wheat straw has been shown by Bey et al. (2011) who found that CDH increased the saccharification process due to gluconic acid production. In this work, we studied the synergism between $\mathrm{CDH}$ and $\mathrm{CBHs}$, EGs, and $\beta$-glucosidases both in vitro and in vivo. The $\mathrm{CDH}$ from Phanerochaete chrysosporium was used to further diminish cellobiose inhibition to cellulases. Supplementation of the purified CDH in Trichoderma cellulase increased the cellulase activity, especially $\beta$-glucosidase activity in vitro. Moreover, the $c d h$ gene was heterologously expressed in T. reesei QM9414 under the control of cbh2 promoter and produced a recombinant version of cellulase mixture. The cellulase production and the hydrolysis efficiency of cdh-expressing Trichoderma transformants were significantly increased.

\section{MATERIALS AND METHODS}

\section{Construction of cdh Expression Vector in P. pastoris}

The $c d h$ gene (GenBank Accession U50409) from Phanerochaete chrysosporium was synthesized in Sangon Biotech (Shanghai, China) and cloned into pUC57, yielding the plasmid pc-cdh.

The native signal peptide and stop codon of the $c d h$ gene were removed by PCR with the forward primer mcdh-Ep1 (EcoR I) and reverse primer mcdh-Xbp2H (Xba I) (Supporting Information Table S1). The plasmid pc-cdh was used as the template. The PCR products were digested with EcoR I and Xba I and inserted into pPICZ $\alpha \mathrm{A}$ at EcoR I and Xba I sites, yielding the plasmid pWM79.

\section{Transformation and Isolation of P. pastoris GS115 Transformants}

The linearized pWM79 was transformed to P. pastoris GS115 by electroporation according to the manufacturer of BTX ECM630 electroporation system (NatureGene Corp.). The vector $\mathrm{pPICZ} \alpha \mathrm{A}$ without insert was used as a control. The transformants were first screened on YPDS plates with different concentrations of zeocin $(200-1000 \mu \mathrm{g} / \mathrm{mL})$, and were further screened in minimal dextrose (MD) plates and methanol (MM) plates according to the Pichia Expression Kit manual (Invitrogen).

Small-scale expression in $20 \mathrm{~mL}$ BMMY medium and following SDS-PAGE and activity assay were used to confirm the recombinant Pichia clones expressing the correct $\mathrm{CDH}$ protein. Zeocin-resistant transformants were inoculated in $10 \mathrm{~mL}$ of BMGY medium (in $50 \mathrm{~mL}$ Erlenmeyer flask) at $28^{\circ} \mathrm{C}$ in a rotary shaker $(200 \mathrm{rpm})$ for $12-16 \mathrm{~h}$ to an OD600 of 2, and the expression was induced by transferring cells into $20 \mathrm{~mL}$ of BMMY growing for a further $96 \mathrm{~h}$. The medium was sampled and 
supplemented with $2 \%(\mathrm{v} / \mathrm{v})$ methanol every $12 \mathrm{~h}$. SDS-PAGE of culture supernatants at different time points was used to identify the optimal induction time and to determine which transformant had the best secretion yield. Scale-up expression was performed in 1 liter Erlenmeyer flask on a rotary shaker $(250 \mathrm{rpm})$ at $28^{\circ} \mathrm{C}$ for $96 \mathrm{~h}$ in $200 \mathrm{~mL}$ medium.

\section{Purification and Characterization of the Recombinant CDH}

After induction for $96 \mathrm{~h}$, the medium was separated from the cells by centrifugation and proceed to purification. The culture supernatant was first concentrated using Amicon Ultra15 (MWCO $30 \mathrm{kD}$, Millipore). The concentrated supernatant was dialyzed against Binding buffer and loaded on a His•Bind Resin, Ni-charged column (Novagen). The purification was according to the HisøBind ${ }^{\circledR}$ Kits manual. The His-tagged $\mathrm{CDH}$ was eluted with Washing Buffer and dialyzed against PBS buffer. The protein was concentrated with Amicon Ultra-15. The concentration of the purified $\mathrm{CDH}$ was measured by the Bradford protein assay kit (Beyotime) with bovine serum albumin (BSA) as standard. The deglycosylation of the $\mathrm{CDH}$ protein was performed by Endo $\mathrm{Hf}$ (NEB).

Cellobiose dehydrogenase activity was determined by monitoring the reduction of $100 \mu \mathrm{M}$ cytochrome $c$ in $50 \mathrm{mM}$ acetate buffer, $\mathrm{pH} 4.5$ containing $1 \mathrm{mM}$ cellobiose. The decrease in absorption at $550 \mathrm{~nm}$ was monitored at $25^{\circ} \mathrm{C}$ for $2 \mathrm{~min}$. The $K_{\mathrm{M}}$ for cellobiose was determined at $25^{\circ} \mathrm{C}$ in $50 \mathrm{mM}$ acetate buffer, $\mathrm{pH} 4.5$ using cytochrome $c$ as electron acceptor. The concentration of cellobiose ranged from 0.004 to $10 \mathrm{mM}$. Graphpad prism 5 was used for the non-linear regression calculation and kinetic parameter determination.

\section{Supplementation of the Purified CDH to Cellulase in vitro}

QM9414 were pre-cultured in $250 \mathrm{~mL}$ Erlenmeyer flasks on a rotary shaker $(200 \mathrm{rpm})$ at $30^{\circ} \mathrm{C}$ for $24 \mathrm{~h}$ in $50 \mathrm{~mL}$ MandelsAndreotti (MA) medium supplemented with $1 \%(\mathrm{w} / \mathrm{v}) \mathrm{D}$ glucose for growth and $0.45 \%(\mathrm{w} / \mathrm{v})$ lactose for initial inducer of cellulase production. Pre-grown mycelia were resuspended in $50 \mathrm{~mL}$ fermentation medium and incubated in $250 \mathrm{~mL}$ Erlenmeyer flasks on a rotary shaker $(200 \mathrm{rpm})$ at $30^{\circ} \mathrm{C}$ for 48, 72, and $96 \mathrm{~h}$. Fermentation medium was composed of MA medium, $1 \%(\mathrm{w} / \mathrm{v}) \mathrm{D}$-glucose and 2.6\% (w/v) lactose (Eventhough glucose is reported to be a repressor of cellulase activities, from optimization experiments, its addition in the medium was contributed to the mycelia growth. The level of residual glucose in the medium reduced to $0.1 \%$ after $24 \mathrm{~h}$ ). Culture supernatants were removed and collected by filtration. The purified $\mathrm{CDH}$ was added to the culture supernatants before cellulase activity analysis and the mixture was incubated at room temperature for $5 \mathrm{~min}$ to disperse uniformly. For comparison of the synergism effects between $\mathrm{CDH}$ and cellulase with the synergism effects between $\beta$-glucosidase and cellulase, the commercial glucosidase from Aspergillus niger (obtained from Sigma-Aldrich) (Ag-Bgl) and a recombinant thermostable $\beta$-glucosidase from Thermatoga maritima (Tm-BglA) (Song et al., 2011) were used to test the synergism effects between $\beta$-glucosidases and Trichoderma cellulase.

\section{Construction of Pcbh2-cdh Expression Cassette}

The plasmid pSMZ1 bearing the promoter and terminator of the cbh2 gene, was generously provided by Professor Christian P. Kubicek from Institute of Chemical Engineering, Vienna University of Technology (Zeilinger et al., 1998). The pSMZ1 was digested with Not I, then blunted and digested again with $X b a$ I to get a blunt/Xba I vector backbone. The plasmid Pccdh containing the $c d h$ gene, was digested with $B a m H \mathrm{I}$, then blunted and digested again with $X b a$ I. The resultant blunt/Xba I $c d h$ fragment was ligated into blunt/Xba I pSMZ1 to yield plasmid pWM78. The Pcbh2-cdh expression cassette, a 4-kbp EcoR I/Hind III fragment from pWM78, was used to transform T. reesei QM9414. Transformation was carried out as described by Gruber et al. (1990) using co-transformation of pSMZ1. Transformants were selected with Hygromycin B resistance and the integration of the $c d h$ gene into the genome was confirmed by genomic PCR. In order to avoid the homologous recombination in cbh2 site, the genome PCR for the cbh2 gene was used to confirm that the $c d h$ expression cassette and Hygromycin B select cassette were not homologously recombined in the genome. The purified transformants were further selected on cellulose Cong Red Medium.

The screened cellulase producers were inoculated in liquid medium. A total of $10^{8}$ conidia per liter were used as inoculum. Mycelia were pre-cultured in $250 \mathrm{~mL}$ Erlenmeyer flasks on a rotary shaker $(200 \mathrm{rpm})$ at $30^{\circ} \mathrm{C}$ for $24 \mathrm{~h}$ in $50 \mathrm{~mL} \mathrm{MA} \mathrm{medium}$ supplemented with $1 \%(\mathrm{w} / \mathrm{v})$ D-glucose for growth and $0.45 \%$ $(\mathrm{w} / \mathrm{v})$ lactose for initial inducer of cellulase production. Pregrown mycelia were resuspended in $50 \mathrm{~mL}$ fermentation medium and incubated in $250 \mathrm{~mL}$ Erlenmeyer flasks on a rotary shaker $(200 \mathrm{rpm})$ at $30^{\circ} \mathrm{C}$ for 48,72 , and $96 \mathrm{~h}$. Fermentation medium was composed of MA medium, 1\% (w/v) D-glucose and 2.6\% $(\mathrm{w} / \mathrm{v})$ lactose. For the experiments using cellulose as carbon source, pre-grown mycelia were resuspended in MA medium supplemented with $1 \%(\mathrm{w} / \mathrm{v})$ microcrystalline cellulose as sole carbon source. The experiments were conducted in triplicate for each sample. After induction, mycelia were collected by filtration, washed, frozen in liquid nitrogen, stored at $-80^{\circ} \mathrm{C}$, and used for total RNA extraction and quantitative real-time PCR analysis. Culture supernatants were used for protein content detection and cellulase activities assays.

\section{RNA Extraction and Transcript Analysis by Quantitative Real-time PCR (qRT-PCR)}

Total RNA was extracted using TRIZOL reagent (Invitrogen, USA) according to the manufacturer's instructions. RNA was quantified by NanoVue (GE Healthcare), and the integrity was checked by gel electrophoresis in $1 \%$ agarose. Total RNA $(1 \mu \mathrm{g})$ from each pooled sample was first digested with DNase I (TaKaRa) to remove genomic DNA. Synthesis of cDNA from total RNA was carried out using a cDNA Synthesis kit (TaKaRa) according to the manufacturer's instructions. First-strand cDNA 
was synthesized from the same amount of total RNA (1 $\mu \mathrm{g})$. Synthesized cDNA was diluted 1:20 and used as a template for quantitative real-time PCR. Reactions were performed in the LightCycler ${ }^{\circledR} 480$ System (Roche) using FastStart Universal SYBR Green Master ROX (Roche) for detection according to the manufacturer's instructions. Actin transcript was used as an internal reference to normalize the amount of total RNA present in each reaction (Verbeke et al., 2009). The fluorescence threshold value was calculated using LightCycler ${ }^{\circledR} 480$ system software. The specificity of the PCR amplifications was documented by melting curve analysis. The expression levels of genes were calculated from the threshold cycle according to the $2^{-\Delta \Delta C T}$ method (Livak and Schmittgen, 2001) relative to transcription levels of QM9414 (Liu et al., 2016). All assays were performed in triplicate with water as a negative control instead of cDNA. Gene-specific primers (Supporting Information Table S1) used in qRT-PCR were designed to amplify $150-200 \mathrm{bp}$ of the internal coding region of each gene. Plasmid cDNA standards are also listed in Supplementary Table S1.

\section{Total Protein Concentration and Biomass Determination}

Total protein concentrations in the culture supernatants were measured by the Bradford protein assay kit (Beyotime) with BSA as standard. For fermentation culture, biomass concentrations were determined by gravimetric analysis. In each time point, mycelia of $20 \mathrm{~mL}$ culture were collected by filtration. The mycelia were washed with $\mathrm{ddH}_{2} \mathrm{O}$ to get rid of salts and filtrated again. The pellets were dried overnight in an oven $\left(70^{\circ} \mathrm{C}\right)$ to constant weight. For cellulose culture, biomass concentrations were indirectly measured by the amount of intracellular protein quantified by the Bradford protein assay kit (Beyotime) with BSA as standard. The results are means of three independent experiments cultivations.

\section{Cellulase Activity Assay}

All samples were analyzed in triplicate and mean values were calculated. Overall cellulase activity of the samples was measured as Filter Paper activities (FPA) using the IUPAC-recommended procedure (Ghose, 1987). Endoglucanase activity was assayed as CMCase activity with carboxymethylcellulase (CMC) as substrate in $50 \mathrm{mM}$ acetate buffer $(\mathrm{pH} 4.8)$ for $15 \mathrm{~min}$ at $50^{\circ} \mathrm{C}$. For both activities, sugar release was assayed via the dinitrosalicylic acid (DNS) method using glucose as the standard. $\beta$-glucosidase activity was determined using 4-nitrophenyl- $\beta$-Dglucopyranoside with paranitrophenol as the standard (Berghem and Pettersson, 1974).

\section{Statistical Analysis}

The statistical significance analyses of all tests were performed with one-way ANOVA followed by the Bonferroni test available in the GraphPad Prism 5.

\section{RESULTS}

\section{Heterologous Expression and Enzymatic Characterization of CDH}

Here the $c d h$ gene from $P$. chrysosporium was chosen as its previous profound investigation. The cDNA encoding $\mathrm{CDH}$ from $P$. chrysosporium has already been cloned by Raices and expressed in P. pastoris KM71 by Yoshida et al. (2001) with the expression vector pPIC9K. In this work, the $c d h$ gene was heterologously expressed in $P$. pastoris GS115 with the expression vector $\mathrm{pPICZ} \alpha \mathrm{A}$. The $c d h$ gene was synthesized and cloned into pUC57, yielding the plasmid pc-cdh.

The TMpred program predicted a membrane-spanning region in the $\mathrm{CDH}$ protein amino acid sequence. SignalP 4.1 program (Petersen et al., 2011) prediction reveals that the location of signal peptide cleavage site is in the 18th amino acid. The expression vector $\mathrm{pPICZ} \alpha \mathrm{A}$ (Invitrogen), containing the $\alpha$-factor secretion signal, allows high-level expression of the target gene in Pichia. In order to construct the $c d h$ gene in pPICZ $\alpha \mathrm{A}$ in frame with the $\alpha$-factor secretion signal and the C-terminal $6 \times \mathrm{His}$ tag, the native signal peptide and the stop codon of the $c d h$ gene were removed by PCR. The digested PCR products were inserted into $\mathrm{pPICZ} \alpha \mathrm{A}$ at the corresponding sites, yielding the plasmid pWM79 (Figure 1A).
A

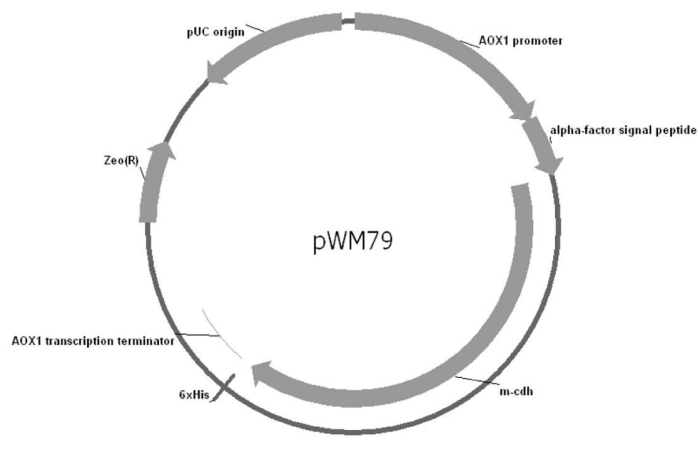

B

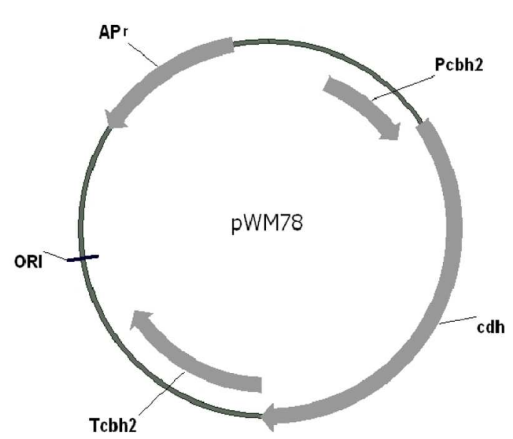

FIGURE 1 | Map of pWM79 and pWM78. (A) Map of the plasmid pWM79 that constructed for transformation of Pichia pastoris GS115. (B) Map of the plasmid pWM78 that constructed for transformation of Trichoderma reesei QM9414. 
The recombinant gene was then introduced into the Pichia genome under the control of the methanol-inducible promoter. Zeocin-resistant transformants were then screened for protein expression. $\mathrm{CDH}$ activity was successfully detected in the supernatant after induction, indicating the functional expression of the $\alpha$-factor signal sequence. The recombinant $\mathrm{CDH}$ was secreted at high levels $(0.3 \mathrm{~g} / \mathrm{L}$ after $96 \mathrm{~h}$ of induction) and was purified using the His-Bind resin.

The purified $\mathrm{CDH}$ displayed a relative molecular weight around $110 \mathrm{kDa}$ appeared on SDS-PAGE (Figure 2A). Western blot analysis confirmed the protein was $\mathrm{CDH}$ (Figure $2 \mathrm{~B}$ ). The protein sequence for possible $N$-glycosylation sites (Asn-XaaSer/Thr) was checked with NetNGlyc 1.0 and six Asn-XaaSer/Thr sites in the sequence were predicted to be $N$-glycosylated. Following deglycosylation of $\mathrm{CDH}$, a band corresponding to its theoretical molecular weight was observed (Figure 2C). The purified $\mathrm{CDH}$ exhibited great affinity for cellobiose, with a $K_{\mathrm{M}}$ value of $8 \mu \mathrm{M}$ and $k_{\text {cat }}$ value of $14.92 \mathrm{~s}^{-1}$.

\section{Synergistic Effects between the Purified CDH with Cellulase of $T$. reesei QM9414}

It has been well known that the expression levels of cellulase genes are different during the time-course cultivation. In order to assess the influence of $\mathrm{CDH}$ addition on the enzymatic hydrolysis of cellulase from $T$. reesei QM9414 at different cultivation time, the filter paper activity (FPA), endoglucanase (CMCase) and $\beta$-glucosidase activity were measured over a range of time points in the presence of 0,10 , and $50 \mu \mathrm{g}$ recombinant $\mathrm{CDH}$.

In the presence of $10 \mu \mathrm{g} \mathrm{CDH}$, a significant increase in FPA activity (approximately 67.7\%, $P<0.05$ ) was observed in the culture supernatant of $48 \mathrm{~h}$ cultivation (Figure 3A), whereas in the presence of $50 \mu \mathrm{g} \mathrm{CDH}$, the increase was not significant

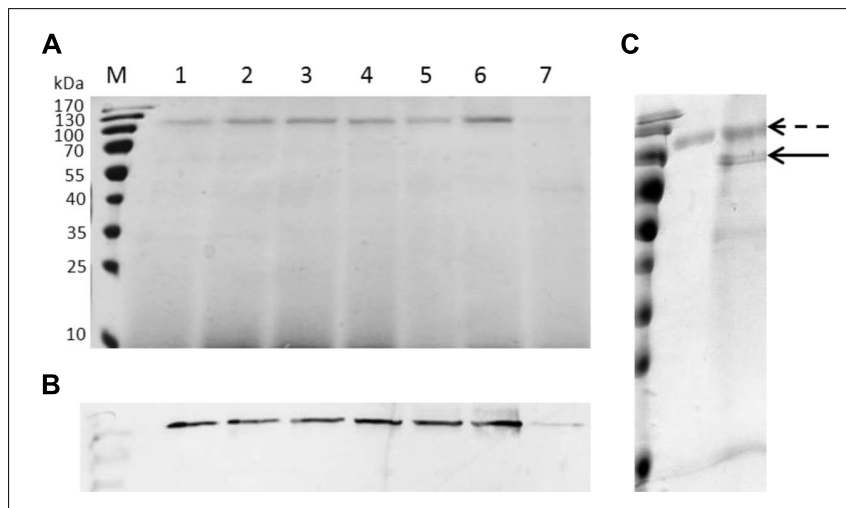

FIGURE 2 | SDS-PAGE, Western blotting analysis and deglycosylation of recombinant CDH. (A) SDS-PAGE. Lane M: protein marker, lane 1-7: the culture supernatants of GS115 harboring pWM78 at 48, 60, 72, 78, 84, 96, $24 \mathrm{~h}$ after induction, respectively. Equal amounts of $10 \mu \mathrm{L}$ samples were loaded for all lanes. At the early stage of induction (lane 7, $24 \mathrm{~h}$ after induction), the amount of protein was $0.2 \mu \mathrm{g}$, while at the late stage of induction (lane 6, $96 \mathrm{~h}$ after induction), the amount of protein was $2.95 \mu \mathrm{g}$. (B) Western blotting analysis using anti-His antibodies. Lanes are the same as A. (C) Deglycosylation of recombinant CDH showed a loss of approximately $30 \mathrm{kDa}$. Dotted arrow indicates $\mathrm{CDH}$ before deglycosylation while solid arrow indicates $\mathrm{CDH}$ after deglycosylation.
A

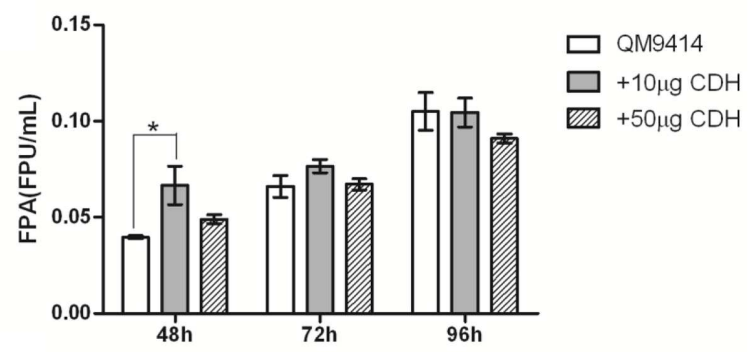

B

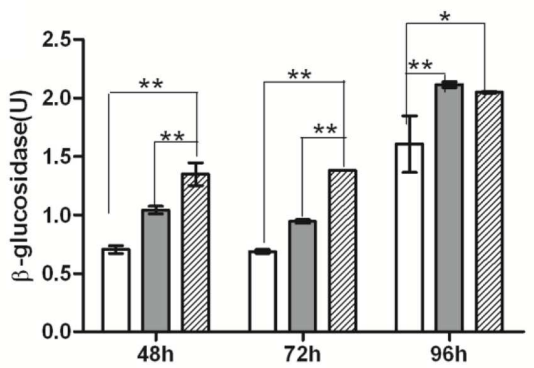

C

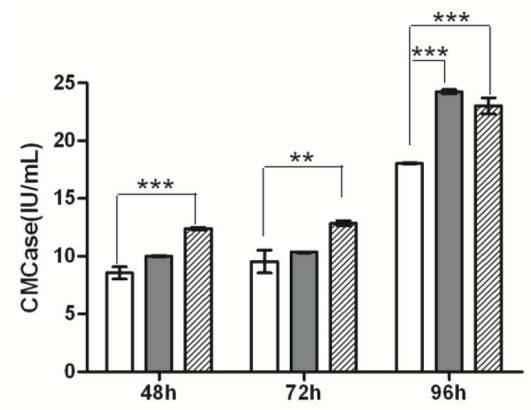

FIGURE 3 | In vitro synergistic effects between the purified CDH and cellulase of $T$. reesei QM9414. FPU activities (A), $\beta$-glucosidase activities (B), and CMCase (C) from the culture supernatants of T. reesei QM9414 for different times as indicated, in the presence of $0 \mu \mathrm{g}$ (QM9414), $10 \mu \mathrm{g}$ $(+10 \mu \mathrm{g} \mathrm{CDH})$ or $50 \mu \mathrm{g}(+50 \mu \mathrm{g} \mathrm{CDH})$ purified $\mathrm{CDH} .{ }^{*} P<0.05,{ }^{* *} P<0.01$, ***P $<0.001$.

( $\sim 23.73 \%, P>0.05)$. For the culture supernatants of 72 and $96 \mathrm{~h}$ cultivation, the changes of FPA activity were slightly increased in the presence of $10 \mu \mathrm{g}$ CDH. In $96 \mathrm{~h}$ culture supernatant, the FPA activity decreased in the presence of $50 \mu \mathrm{g} \mathrm{CDH}$. As for CMCase, the addition of $10 \mu \mathrm{g} \mathrm{CDH}$ caused approximately $8-34 \%$ increased activity at different time points (Figure 3C). When loading $50 \mu \mathrm{g} \mathrm{CDH}$ it was increased to approximately $27-45 \%$. $\beta$-glucosidases seem to be more influenced by $\mathrm{CDH}$ addition. In the presence of $10 \mu \mathrm{g} \mathrm{CDH}, \beta$-glucosidase activities were approximately $48,37.5$, and $32 \%$ higher in the culture supernatant of 48, 72, and $96 \mathrm{~h}$ cultivation (Figure 3B), respectively. As expected, further increasing $\mathrm{CDH}$ addition to $50 \mu \mathrm{g}$ increased approximately 91 and 100\% $\beta$-glucosidase activity in the culture supernatant of 48 and $72 \mathrm{~h}$ cultivation. In the culture supernatant of $96 \mathrm{~h}$ cultivation, $50 \mu \mathrm{g} \mathrm{CDH}$ 
addition did not significantly further increase either CMCase or $\beta$-glucosidase compared to the enzyme dosage of $10 \mu \mathrm{g} \mathrm{CDH}$ supplementation.

It indicates that the influence of $\mathrm{CDH}$ supplementation on cellulase not only depends on the $\mathrm{CDH}$ dosage, but also on the cultivation times of $T$. reesei QM9414, which correspond to different dosage and composition of cellulase mixture.

In the presence of $10 \mu \mathrm{g} \mathrm{Ag-Bgl,} \mathrm{approximately} 15.2-17.6 \%$ increase in FPA activity were observed in the culture supernatants of 48 and 72-h cultivations (Supplementary Figure S1). For the culture supernatants of 96-h cultivation, the FPA activity were increased 9\% in the presence of $10 \mu \mathrm{g}$ Ag-Bgl. Consideration with the $0.038 \%$ residue glucose in the culture supernatant of 48 -h point, the limited synergism effects between $\beta$-glucosidases and cellulase at 48 -h point were due to the product inhibition of $\beta$-glucosidase by glucose. As for CMCase, the addition of $10 \mu \mathrm{g}$ Ag-Bgl or Tm-BglA showed similar increased activity with $\mathrm{CDH}$ at different time points.

\section{In Vivo Synergistic Effects between Heterologous CDH and Native Cellulase in T. reesei QM9414}

As the results showed above, the addition of recombinant $\mathrm{CDH}$ to a reaction mixture containing $T$. reesei QM9414 cellulase increased FPA, CMCase, and $\beta$-glucosidase activities. To discover how the synergy is functional in vivo, we then expressed the $c d h$ in T. reesei QM9414 and evaluated the in vivo synergistic effects between $\mathrm{CDH}$ and cellulase. The promoter of the cbh2 gene of T. reesei was used and the plasmid pWM78 (Figure 1B) was constructed as described in Materials and Methods. The transformant with the best performance was named as cdh:: QM9414. To guarantee comparisons between the native and mutant strains, we examined the growth pattern that was represented by dry weight biomass. The cdh::QM9414 showed retarded but no significant difference in growth when comparing to the parental QM9414 (Supplementary Figure S2).

The cellulase activity (FPA, CMCase, and $\beta$-glucosidase) were measured from the culture mediums of the wild-type and the mutant strains and the results were shown in Figure 4. The cdh::QM9414 had higher cellulase activities compared with the parental QM9414. The FPA activities were 47-59\% higher at different cultivation time points. $\beta$-glucosidase activities were increased considerably by 10.5 and 2.4 times while CMCase activities were increased 70 and $100 \%$ at 72 and $96 \mathrm{~h}$, respectively.

The vast majority of the proteins in the culture supernatant of T. reese $i$ is cellulase, especially three major cellulase components (CBH I, CBH II, and EG II). To determine whether the increased cellulase activity was also accompanied with the changes in cellulase expression level compared with parental QM9414, we analyzed the extracellular proteins of the $c d h$-expressing and the native QM9414 strains by SDS-PAGE (Supplementary Figure S3), and the secreted proteins were quantified by Bradford assay. Through the whole cultivation process the expression of $c d h$ enriched the cellulase production compared with QM9414. This did not correlate with the growth profile (Supplementary Figure
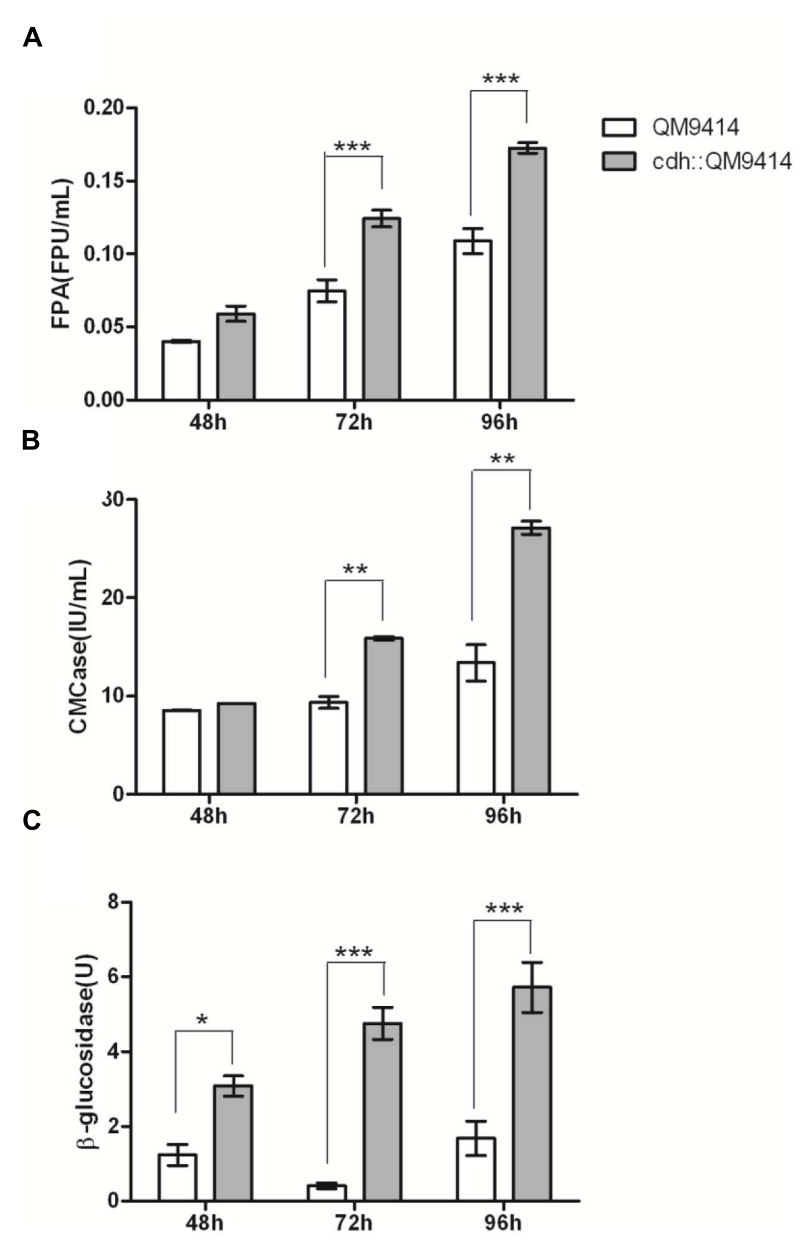

FIGURE 4 | In vivo synergistic effects between $\mathrm{CDH}$ and cellulase of T. reesei QM9414. FPA activities (A), CMCase activities (B), and $\beta$-glucosidase activities (C) from the culture supernatants of T. reesei QM9414 and cdh::QM9414 for different times as indicated. Error bars are represented from three biological replicates. ${ }^{*} P<0.05,{ }^{* *} P<0.01,{ }^{* * *} P<0.001$.

S2). Bradford assay showed the increase was 73\% $(P<0.001)$ higher at 48 and $26.4 \%(P<0.001)$ higher at $96 \mathrm{~h}$. The BGL1 production, in particular, was remarkably enriched in the $c d h$-expressing strain. It is commonly accepted that the formation of cellulase is regulated at transcriptional level. This observation motivated us to investigate if the mRNA level of bgll gene was indeed positively elevated. As the maximum increase of $\beta$-glucosidase activity was observed in $72 \mathrm{~h}$, and the enhancement of BGL1 production was obvious in $48 \mathrm{~h}$, we detected the mRNA levels in $48 \mathrm{~h}$. Total RNA was extracted from mycelia after $48 \mathrm{~h}$ growing, from which cDNA was prepared and subjected to qRT-PCR (Figure 5). In the $c d h$ expressing strain cdh:: QM9414, mRNA level for bgl1 gene was 2.3 times higher than level in native T. reesei QM9414. This is consistent with the protein levels as judged on SDS-PAGE gel (Supplementary Figure S3), but much lower than the 10.5-time increase observed in $\beta$-glucosidase activity (Figure 4C). This suggested that the enhancement of cellulase activity caused by 


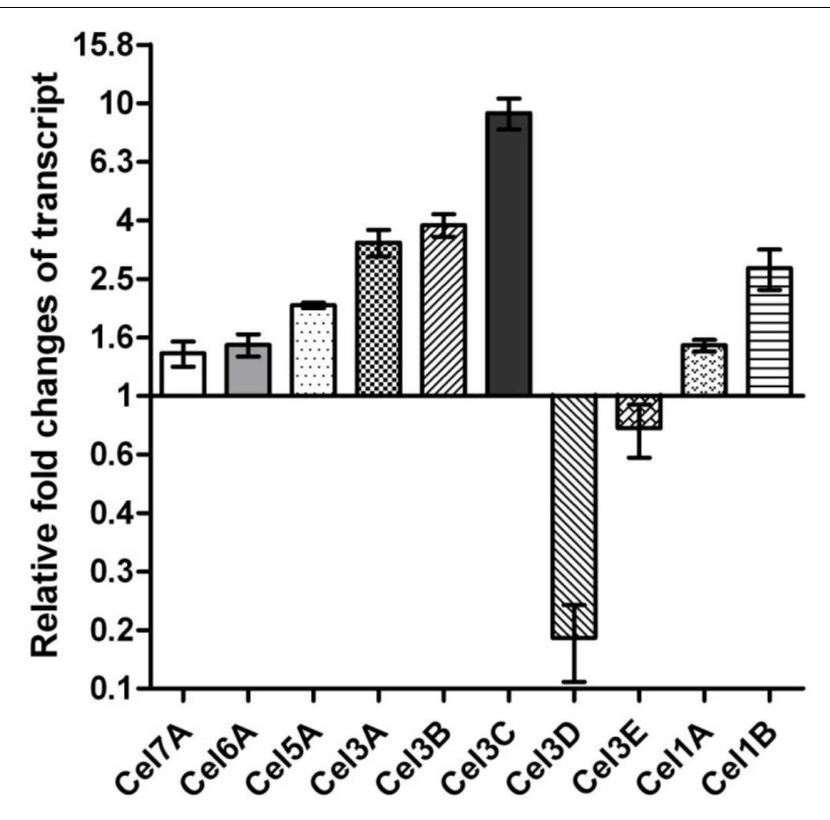

FIGURE 5 | Transcriptional changes of the selected genes in cdh-expressing strain compared to parental QM9414.

$\mathrm{CDH}$ is not only due to its indirect effects on inducement of enzyme production, but also a functional synergism with cellulase during hydrolysis.

Eleven $\beta$-glucosidases have so far been characterized or predicted in $T$. reese $i$ according to the predictions in the genome database (T. reesei v2.0) and CAZy database (Martinez et al., 2008). Besides the bgll, we also wondered whether there is an increased up-regulation of other $\beta$-glucosidase genes of cdh::QM9414. We further detected relative mRNA levels of other six $\beta$-glucosidase (CEL1A, CEL1B, CEL3A, CEL3B, CEL3C, CEL3D, CEL3E) genes with qRT-PCR. Four of the six genes displayed the increased transcript levels. Relatively high levels of expression of these $\beta$-glucosidase genes in $c d h$-expressing strain implied that it might be caused by cellobiose degradation.

\section{In Vivo Synergistic Effects between CDH and Cellulase with Cellulose as the Carbon Source}

As the results showed above, the synergism between $\mathrm{CDH}$ and cellulase was observed in vivo in fermentation process with lactose as the soluble carbon source. To get a better insight into the synergistic effects on natural substrates, we examined the performance of the $c d h$ transformant strain with cellulose as the carbon source. The cdh::QM9414 and the parental QM9414 were pre-cultured in MA media supplemented with $0.45 \%$ $(\mathrm{w} / \mathrm{v})$ lactose and resuspended in MA media containing 1\% (w/v) microcrystalline cellulose as the sole carbon source. As the insoluble cellulose particles were enwrapped with mycelia, it is hard to weigh the dry biomass. Thus the amount of intracellular protein content was measured for monitoring cell growth. No difference was observed on cellulose at 48 and $72 \mathrm{~h}$
(Supplementary Figure S4A). At $96 \mathrm{~h}$, the intracellular protein of cdh::QM9414 decreased about 50\% compared to the parental QM9414 strain.

The extracellular protein content was determined by Bradford assay and the expression profile was analyzed on SDS-PAGE (Supplementary Figure S5). When growing on cellulose, the enzyme production of cdh::QM9414 was increased by $10-25 \%$ at different time points compared to QM9414 (Supplementary Figure S4B). The abundance of the expressed $\mathrm{CDH}$ and BGL1 proteins are much lower than the three major cellulase components (Supplementary Figure S5). With cellulose as the carbon source, the cdh::QM9414 also secreted CDH protein into the culture medium, and enhanced the BGL1 production. Compared with the expression profile on lactose as the carbon source (Supplementary Figure S3), the cellulase was notably induced by cellulose. In addition, although the $c d h$ was controlled under the $c b h 2$ promoter, the protein expression level for $\mathrm{CDH}$ production is far behind that achievable by native $\mathrm{CBH}$ II (Supplementary Figure S5).

Different from the synergism observed with lactose as the carbon source, the most significant synergism between $\mathrm{CDH}$ and cellulase components was observed in FPA catalysis rather than $\beta$-glucosidase (Figure 6). The FPA activities of cdh::QM9414 were increased about 2.5-3.8 times compared to QM9414 on cellulose. This was not corresponding with the protein expression profile on SDS-PAGE. Therefore, there is also a functional synergism between $\mathrm{CDH}$ and cellulase during hydrolysis of cellulose. The CMCase activities were raised sharply at $48 \mathrm{~h}$ on cellulose, but slowed down at 72 and $96 \mathrm{~h}$. $\beta$-glucosidase activities increased 190 and $39 \%$ at $48 \mathrm{~h}$ and $72 \mathrm{~h}$, respectively, whereas there is no significant change compared to parent strain QM9414 at $96 \mathrm{~h}$ (Figure 6). It's confirmed that a $c d h$-expressing T. reesei strain has a synergistic effect upon the overall cellulase activity on pure microcrystalline cellulose.

\section{DISCUSSION}

Currently cellulosic biofuels production receives massive attention (Fairley, 2011). However, efficient cellulase production has been a hurdle to be overcome. Although the model cellulolytic organism $T$. reesei is presently used to produce cellulase mixtures, the productivity and the performance for some prohibitive substrates require further improvement for such industrial enzyme-based production system to make it economically viable (Klein-Marcuschamer et al., 2012). Enzymatic hydrolysis of cellulose is a complex process, where the last step is a critical step and involves a catalytic reaction of $\beta$-glucosidase with cellobiose as the substrate, a strong inhibitor of both cellobiohydrolases and endocellulases. The native $\beta$-glucosidases produced by Trichoderma are not enough to reduce cellobiose inhibition and additional $\beta$-glucosidase supplementation is necessary. Research efforts on cellulase performance have recently widened scope to explore new types of enzymes that substantially improve cellulose degradation. Synergistic interactions of different enzymes or non-enzymatic proteins result in more efficient cellulose degradation. The 
A

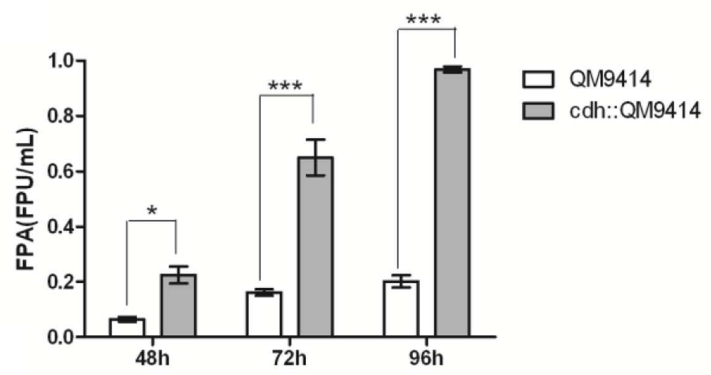

B

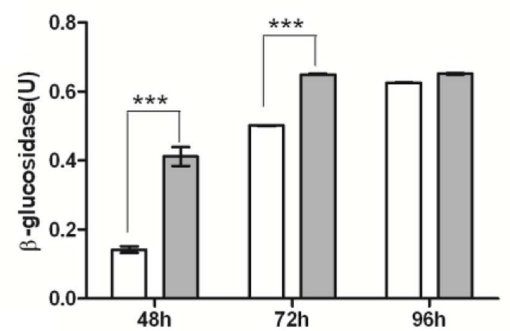

C

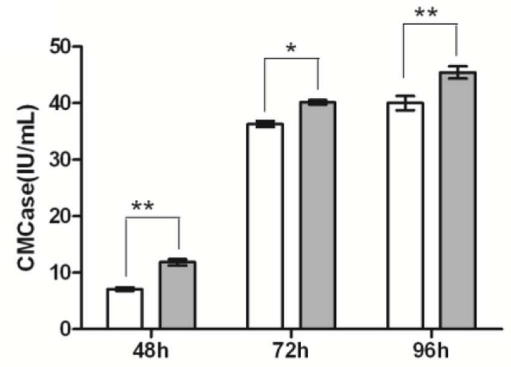

FIGURE 6 | In vivo synergistic effects between $\mathrm{CDH}$ and cellulase of T. reesei QM9414 with cellulose as carbon source. FPA activities (A), $\beta$-glucosidase activities (B), and CMCase activities (C) from the culture supernatants of T. reesei QM9414 and cdh::QM9414 with cellulose as carbon source for different times as indicated. Error bars are represented from three biological replicates. ${ }^{*} P<0.05,{ }^{* *} P<0.01,{ }^{* * *} P<0.001$.

oxidative enzyme AA9 (formerly GH61, reclassified as family AA9 by CAZy, Lombard et al., 2014) could promote the efficiency of classical cellulases (Horn et al., 2012), and the oxidative enzymes are now present in commercially available cellulase preparations such as $\mathrm{CTec} 2$ to improve the conversion yield (Cannella et al., 2012). CDH was also reported as oxidative enzyme. In this study, we explored the synergistic effects between cellulase and $\mathrm{CDH}$, and discuss the promising of combining $\mathrm{CDH}$ with the current $\beta$-glucosidase and other available addition enzymes to enhance cellulase performance.

Although the physiological function of $\mathrm{CDH}$ in cellulose biodegradation has not been elucidated yet, some experimental observations related to a possible function have been made: $\mathrm{CDH}$ enhances $P$. chrysosporium $\mathrm{CBH}$ I activity by oxidizing the inhibitor cellobiose to cellobionolactone. In the current study, we confirmed the synergism between the purified $\mathrm{CDH}$ and cellulase from $T$. reesei in vitro and further constructed a $c d h$ overexpressed stain and investigated the synergism in vivo. Based on both in vitro and in vivo experiments, the synergism was illustrated with FPA, CMCase and $\beta$-glucosidase activities. Besides the synergism between $\mathrm{CDH}$ and $\mathrm{CBH}$ I that observed previously, we found that $\mathrm{CDH}$ increased the $\beta$-glucosidase activity remarkably. It implies that $\mathrm{CDH}$ and $\beta$-glucosidases work synergistically in cellobiose metabolism. However, the comparison of synergism effects between CDH and cellulase with synergism effects between $\beta$-glucosidases and cellulase indicates different functions of $\mathrm{CDH}$ and $\beta$-glucosidases during the enzymatic hydrolysis process. The supplemention of $\beta$-glucosidases mainly balances the deficiency of low amount of $\beta$-glucosidases in $T$. reesei cellulase and releases the inhibition of the accumulation of cellobiose as the end product. And $\beta$-glucosidases are also sensitive to the product inhibition by glucose. Combined with the transcriptional analysis of $b g l$ and $c d h$ in relation to cellobiose metabolism (Yoshida et al., 2004) some light could be thrown on how intracellular expression of $\mathrm{CDH}$ lead to an increase in the transcript levels of $\beta$-glucosidase. Transcriptional analysis in $T$. reesei has revealed that the transcription of bgl gene is repressed by cellobiose. It is reasonable to assume that the expression of $\mathrm{CDH}$ decrease the cellobiose concentration and relieve inhibition of $\beta$-glucosidase transcription. However, a more systematic insight into the mechanism with the exploration of the synergistic dynamics and interactions between $\mathrm{CDH}$ and $\mathrm{CBH}$ I, CBH II, EGs, and $\beta$-glucosidases need to be elucidated in future.

The diversity of $\beta$-glucosidases transcription suggests that these enzymes are regulated and functioning in different ways. Although BGL1, CEL3B, and CEL3E all belongs to the family GH3 and are predicted to be secreted according to the signal sequence prediction (SignalP 4.0), but their transcriptions are different. CEL3C and CEL3D, which are in the same protein homology cluster showed similar results. Both amino acid sequences showed no predicted transmembrane domain or signal peptide, which in turn means that both CEL3C and CEL3D work in the cytoplasm. Notably, CEL3C was up-regulated with 9.27-fold changes. This result confirmed the different functional subgroups of GH3 family determined according to phylogenetic analysis (Hakkinen et al., 2012) (Supplementary Figure S6).

The expression of $c d h$ was also found to cause a slightly increased mRNA level of $c b h 1, c b h 2$, and eg2 than those in the native $T$. reesei. One possible explanation is that the expression of the different cellulase genes has been reported to be coordinative. Continuing interest and efforts in understanding the regulating of cellulase has revealed that the expression of $c b h 1, c b h 2$, and eg2 genes are subjected coordinatly to both positive (Xyrl, Ace2, Hap2/3/5) and negative (Crel, Acel) regulation (Schmoll and Kubicek, 2003). The recent exploration of the bgll, cella, and cellb in the induction of cellulase genes by lactose in $T$. reesei demonstrated that $c b h 1$ gene expression is co-regulated with $\beta$-glucosidase genes ( $\mathrm{Xu}$ et al., 2014). The intracellular $\beta$-glucosidase also acts on activating XYR1, which in turns regulates the $c b h 1, c b h 2$, and eg2 gene expression. 
Cellobiose dehydrogenase oxidizes cellobiose to cellobionolactone, which is subsequently hydrolyzed to cellobionic acid. In $P$. chrysosporium, $\mathrm{CDH}$ increased $\mathrm{CBH}$ I activity by releasing competitive inhibition on $\mathrm{CBH}$ I by oxidization of cellobiose to cellobionolactone, however, it is reported that cellobionolactone was more inhibitory to the T. viride CBH I (Igarashi et al., 1998). On the other hand, though cellobionic acid is known to be less inhibitory for cellulases than cellobiose (Igarashi et al., 1998), it could be hydrolyzed by $\beta$-glucosidase at a very low rate (almost 10 -fold lower than for cellobiose), and the formed gluconic acid showed stronger product inhibition than glucose (Cannella et al., 2012). In this study, particularly shown in Figure $\mathbf{3 A}$, when extra $\mathrm{CDH}$ (50 $\mu \mathrm{g}$ vs. $10 \mu \mathrm{g}$ ) was added to cellulase mixture, a decrease of the FPA activity was observed. The mixture of cellulase and $\mathrm{CDH}$ will require further tuning to obtain the optimal radio and enzyme dose to maximize their cellulolytic synergism function.

The $\mathrm{CDH}$ expression in $T$. reesei needs to be optimized further. In consideration of the conclusion derived from Figure 3, we constructed the plasmid with the $c d h$ under the relatively mild cbh 2 promoter instead of commonly used strong promoter cbh1. Unexpectedly, with the same promoter $c b h 2$, the protein expression level for $c d h$ production is far behind that achievable by native $\mathrm{CBH}$ II. Thus the $\mathrm{CDH}$ expression needs to be enhanced by using other promising promoters such as cbhl. Here we chose QM9414 as the parental strain and next this strategy could also be applied to the industrial strains. One of the highest cellulase producers is RUT-C30 with a titer of $30 \mathrm{~g} / \mathrm{L}$ and a number of industrial strains are derived from this mutant (Seidl et al., 2008; Kubicek et al., 2009; Schuster et al., 2012). Further exploration of the synergism in RUT-C30 or other derived industrial strains should be very important. Another notable aspect is the development of new pretreatment procedures for the improved cellulose digestibility. Various types of pretreatment procedures result in different cellulose accessibilities. Thus, in real industrial processing, the impact of adding $\mathrm{CDH}$ to cellulase cocktails is likely to vary depending on the substrates. Our results only showed the synergism with soluble lactose and pure microcrystalline cellulose as carbon source. More work is needed to further refine the synergism with more complex carbon sources.

\section{REFERENCES}

Ayers, A. R., Ayers, S. B., and Eriksson, K. E. (1978). Cellobiose oxidase, purification and partial characterization of a hemoprotein from Sporotrichum pulverulentum. Eur. J. Biochem. 90, 171-181. doi: 10.1111/j.1432-1033.1978.tb12588.x

Bao, W. J., and Renganathan, V. (1992). Cellobiose oxidase of Phanerochaete chrysosporium enhances crystalline cellulose degradation by cellulases. FEBS Lett. 302, 77-80. doi: 10.1016/0014-5793(92)80289-s

Berghem, L. E. R., and Pettersson, L. G. (1974). Mechanism of enzymatic cellulose degradation - isolation and some properties of a $\beta$-glucosidase from Trichoderma viride. Eur. J. Biochem. 46, 295-305. doi: 10.1111/j.14321033.1974.tb03621.x

Bey, M., Berrin, J. G., Poidevin, L., and Sigoillot, J. C. (2011). Heterologous expression of Pycnoporus cinnabarinus cellobiose dehydrogenase in Pichia
In summary, this study demonstrated synergistic effects between $\mathrm{CDH}$ and $T$. reesei cellulase components including FPA, CMCase, and $\beta$-glucosidase with both in vitro and in vivo observations. The results not only confirmed the synergism between $\mathrm{CDH}$ and $P$. chrysosporium $\mathrm{CBH} \mathrm{I}$ that previously observed, but also suggested that $\mathrm{CDH}$ increased the $\beta$-glucosidase activity remarkably. It implies that $\mathrm{CDH}$ and $\beta$-glucosidases work synergistically in cellobiose metabolism. It elucidates a possible mechanism for diminishing the cellobiose inhibition on cellulases by $\mathrm{CDH}$. An integration of different strategies including $\mathrm{CDH}$ expression shows a promising potential for improving cellulose efficiency and thus promoting cellulosic biofuels production.

\section{AUTHOR CONTRIBUTIONS}

MW and XL designed and coordinated the study and wrote the manuscript. MW carried out the experiments and analyzed the results. Both authors read and approved the final manuscript.

\section{FUNDING}

This work was supported by the National Natural Science Foundation of China (NSFC, Grant No. 31100585) and a Basic Research Project of Qingdao (12-1-4-9-(1)-jch) funded by the Qingdao Municipal Science and Technology Commission.

\section{ACKNOWLEDGMENTS}

We thank Professor Christian P. Kubicek and Dr. Susanne Zeilinger-Migsich (Institute of Chemical Engineering, Vienna University of Technology) for kindly providing PSMZ1 plasmid.

\section{SUPPLEMENTARY MATERIAL}

The Supplementary Material for this article can be found online at: http://journal.frontiersin.org/article/10.3389/fmicb. 2016.00620

pastoris and involvement in saccharification processes. Microb. Cell Fact. 10, 113. doi: 10.1186/1475-2859-10-113

Cannella, D., Hsieh, C. W. C., Felby, C., and Jorgensen, H. (2012). Production and effect of aldonic acids during enzymatic hydrolysis of lignocellulose at high dry matter content. Biotechnol. Biofuels 5, 26. doi: 10.1186/1754-68345-26

Dashtban, M., and Qin, W. S. (2012). Overexpression of an exotic thermotolerant $\beta$-glucosidase in Trichoderma reesei and its significant increase in cellulolytic activity and saccharification of barley straw. Microb. Cell Fact. 11, 63. doi: 10.1186/1475-2859-11-63

Fairley, P. (2011). Introduction: next generation biofuels. Nature 474, S2-S5. doi: $10.1038 / 474 \mathrm{~S} 02 \mathrm{a}$

George, S. P., Ahmad, A., and Rao, M. B. (2001). Studies on carboxymethyl cellulase produced by an alkalothermophilic actinomycete. Bioresour. Technol. 77, 171-175. doi: 10.1016/S0960-8524(00)00150-4 
Ghose, T. K. (1987). Measurement of cellulase activities. Pure Appl. Chem. 59, 257-268. doi: 10.1351/pac198759020257

Gruber, F., Visser, J., Kubicek, C. P., and Degraaff, L. H. (1990). Cloning of the Trichoderma reesei pyrG gene and its use as a homologous marker for a high-frequency transformation system. Curr. Genet. 18, 447-451. doi: 10.1007/bf00309915

Gusakov, A. V., Salanovich, T. N., Antonov, A. I., Ustinov, B. B., Okunev, O. N., Burlingame, R., et al. (2007). Design of highly efficient cellulase mixtures for enzymatic hydrolysis of cellulose. Biotechnol. Bioeng. 97, 1028-1038. doi: 10.1002/bit.21329

Hakkinen, M., Arvas, M., Oja, M., Aro, N., Penttila, M., Saloheimo, M., et al. (2012). Re-annotation of the CAZy genes of Trichoderma reesei and transcription in the presence of lignocellulosic substrates. Microb. Cell Fact. 11, 134. doi: 10.1186/1475-2859-11-134

Henriksson, G., Johansson, G., and Pettersson, G. (2000). A critical review of cellobiose dehydrogenases. J. Biotechnol. 78, 93-113. doi: 10.1016/S01681656(00)00206-6

Holtzapple, M., Cognata, M., Shu, Y., and Hendrickson, C. (1990). Inhibition of Trichoderma reesei cellulase by sugars and solvents. Biotechnol. Bioeng. 36, 275-287. doi: 10.1002/bit.260360310

Horn, S. J., Vaaje-Kolstad, G., Westereng, B., and Eijsink, V. G. H. (2012). Novel enzymes for the degradation of cellulose. Biotechnol. Biofuels 5, 45. doi: $10.1186 / 1754-6834-5-45$

Igarashi, K., Samejima, M., and Eriksson, K. E. L. (1998). Cellobiose dehydrogenase enhances Phanerochaete chrysosporium cellobiohydrolase I activity by relieving product inhibition. Eur. J. Biochem. 253, 101-106. doi: 10.1046/j.14321327.1998.2530101.x

Ju, L. K., and Afolabi, O. A. (1999). Wastepaper hydrolysate as soluble inducing substrate for cellulase production in continuous culture of Trichoderma reesei. Biotechnol. Prog. 15, 91-97. doi: 10.1021/bp980116n

Klein-Marcuschamer, D., Oleskowicz-Popiel, P., Simmons, B. A., and Blanch, H. W. (2012). The challenge of enzyme cost in the production of lignocellulosic biofuels. Biotechnol. Bioeng. 109, 1083-1087. doi: 10.1002/bit. 24370

Knauf, M., and Moniruzzaman, M. (2004). Lignocellulosic biomass processing: a perspective. Int. Sugar J. 106, 147-150.

Kubicek, C. P., Mikus, M., Schuster, A., Schmoll, M., and Seiboth, B. (2009). Metabolic engineering strategies for the improvement of cellulase production by Hypocrea jecorina. Biotechnol. Biofuels 2, 19. doi: 10.1186/1754-6834-2-19

Liu, K. M., Dong, Y. M., Wang, F. Z., Jiang, B. J., Wang, M. Y., and Fang, X. (2016). Regulation of cellulase expression, sporulation, and morphogenesis by velvet family proteins in Trichoderma reesei. Appl. Microbiol. Biotechnol. 100, 769-779. doi: $10.1007 / \mathrm{s} 00253-015-7059-2$

Livak, K. J., and Schmittgen, T. D. (2001). Analysis of relative gene expression data using real-time quantitative PCR and the $2-\Delta \Delta$ CT method. Methods 25, 402-408. doi: 10.1006/meth.2001.1262

Lombard, V., Golaconda Ramulu, H., Drula, E., Coutinho, P. M., and Henrissat, B. (2014). The carbohydrate-active enzymes database (CAZy) in 2013. Nucleic Acids Res. 42, D490-D495. doi: 10.1093/nar/gkt1178

Ma, L., Zhang, J., Zou, G., Wang, C. S., and Zhou, Z. H. (2011). Improvement of cellulase activity in Trichoderma reesei by heterologous expression of a $\beta$-glucosidase gene from Penicillium decumbens. Enzyme Microb. Technol. 49, 366-371. doi: 10.1016/j.enzmictec.2011.06.013

Martinez, D., Berka, R. M., Henrissat, B., Saloheimo, M., Arvas, M., Baker, S. E., et al. (2008). Genome sequencing and analysis of the biomass-degrading fungus Trichoderma reesei (syn. Hypocrea jecorina). Nat. Biotechnol. 26, 553-560. doi: $10.1038 /$ nbt1403

Nakazawa, H., Kawai, T., Ida, N., Shida, Y., Kobayashi, Y., Okada, H., et al. (2012). Construction of a recombinant Trichoderma reesei strain expressing Aspergillus aculeatus $\beta$-glucosidase 1 for efficient biomass conversion. Biotechnol. Bioeng. 109, 92-99. doi: 10.1002/bit.23296

Nummi, M., Nikupaavola, M. L., Lappalainen, A., Enari, T. M., and Raunio, V. (1983). Cellobiohydrolase from Trichoderma reesei. Biochem. J. 215, 677-683. doi: 10.1042/bj2150677

Park, J. I., Steen, E. J., Burd, H., Evans, S. S., Redding-Johnson, A. M., Batth, T., et al. (2012). A thermophilic ionic liquid-tolerant cellulase cocktail for the production of cellulosic biofuels. PLoS ONE 7:e37010. doi: 10.1371/journal.pone.0037010

Petersen, T. N., Brunak, S., von Heijne, G., and Nielsen, H. (2011). SignalP 4.0: discriminating signal peptides from transmembrane regions. Nat. Methods 8, 785-786. doi: 10.1038/nmeth.1701

Schmoll, M., and Kubicek, C. P. (2003). Regulation of Trichoderma cellulase formation: lessons in molecular biology from an industrial fungus. Acta Microbiol. Immunol. Hung. 50, 125-145. doi: 10.1556/amicr.50.2003.2-3.3

Schuster, A., Bruno, K. S., Collett, J. R., Baker, S. E., Seiboth, B., Kubicek, C. P., et al. (2012). A versatile toolkit for high throughput functional genomics with Trichoderma reesei. Biotechnol. Biofuels 5, 1. doi: 10.1186/1754-6834-5-1

Seidl, V., Gamauf, C., Druzhinina, I. S., Seiboth, B., Hartl, L., and Kubicek, C. P. (2008). The Hypocrea jecorina (Trichoderma reesei) hypercellulolytic mutant RUT C30 lacks a $85 \mathrm{~kb}$ (29 gene-encoding) region of the wild-type genome. BMC Genomics 9:327. doi: 10.1186/1471-2164-9-327

Song, X. F., Xue, Y. M., Wang, Q. L., and Wu, X. X. (2011). Comparison of three thermostable beta-glucosidases for application in the hydrolysis of soybean isoflavone glycosides. J. Agric. Food Chem. 59, 1954-1961. doi: $10.1021 /$ jf1046915

Verbeke, J., Coutinho, P., Mathis, H., Quenot, A., Record, E., Asther, M., et al. (2009). Transcriptional profiling of cellulase and expansin-related genes in a hypercellulolytic Trichoderma reesei. Biotechnol. Lett. 31, 1399-1405. doi: 10.1007/s10529-009-0030-5

Xu, J., Zhao, G., Kou, Y., Zhang, W., Zhou, Q., Chen, G., et al. (2014). Intracellular $\beta$-glucosidases CEL1a and CEL1b are essential for cellulase induction on lactose in Trichoderma reesei. Eukaryot. Cell 13, 1001-1013. doi: 10.1128/ec.00100-14

Yoshida, M., Igarashi, K., Kawai, R., Aida, K., and Samejima, M. (2004). Differential transcription of $\beta$-glucosidase and cellobiose dehydrogenase genes in cellulose degradation by the basidiomycete Phanerochaete chrysosporium. FEMS Microbiol. Lett. 235, 177-182. doi: 10.1016/j.femsle.2004.04.032

Yoshida, M., Ohira, T., Igarashi, K., Nagasawa, H., Aida, K., Hallberg, B. M., et al. (2001). Production and characterization of recombinant Phanerochaete chrysosporium cellobiose dehydrogenase in the methylotrophic yeast Pichia pastoris. Biosci. Biotechnol. Biochem. 65, 2050-2057. doi: 10.1271/bbb.65.2050

Zeilinger, S., Mach, R. L., and Kubicek, C. P. (1998). Two adjacent protein binding motifs in the cbh2 (cellobiohydrolase II-encoding) promoter of the fungus Hypocrea jecorina (Trichoderma reesei) cooperate in the induction by cellulose. J. Biol. Chem. 273, 34463-34471. doi: 10.1074/jbc.273.51.34463

Zhang, Y. H., and Lynd, L. R. (2004). Toward an aggregated understanding of enzymatic hydrolysis of cellulose: noncomplexed cellulase systems. Biotechnol. Bioeng. 88, 797-824. doi: 10.1002/bit.20282

Conflict of Interest Statement: The authors declare that the research was conducted in the absence of any commercial or financial relationships that could be construed as a potential conflict of interest.

Copyright $\odot 2016$ Wang and Lu. This is an open-access article distributed under the terms of the Creative Commons Attribution License (CC BY). The use, distribution or reproduction in other forums is permitted, provided the original author(s) or licensor are credited and that the original publication in this journal is cited, in accordance with accepted academic practice. No use, distribution or reproduction is permitted which does not comply with these terms. 